\title{
Response to combination of pembrolizumab and axitinib in Hereditary Leyomiomatosis and Renal Cell Cancer (HLRCC)
}

\author{
Ibon Gurruchaga Sotés ${ }^{1^{*}}$, Carmen Santander Lobera ${ }^{2}$ \\ 1* Medical Oncology Department I Miguel Servet University Hospital; gurrutxaga.ibon@gmail.com \\ 2 Medical Oncology Department I Miguel Servet University Hospital; csantlob@yahoo.es
}

\begin{abstract}
Mutations at the gene of fumarate hydratase in which both alleles are affected leads to premature death, however if it present in one allele patient could develop Hereditary Leyomiomatosis an Renal Cell Cancer, a rare disease associated with cutaneous piloleiomyomas and type II papillary renal cell carcinoma. Treatment of this tumor is scarce nowadays, with high mortality in those cases suffering metastatic disease. In clinical guidelines, such as National Comprehensive Cancer Network guidelines, it is embodied as first therapeutic option the combination of erlotinib and bevacizumab, based on a small clinical trial which included patients with HLRCC. With this case report we bring out another treatment option, not described before, with extraordinary results based on the combination of a check-point inhibitor and a tyrosine-kinase inhibitor, based on theoretical tumor immunogenic environment.
\end{abstract}

Keywords: Hereditary Leyomiomatosis and Renal Cell Cancer; Pembrolizumab; Axitinib

\section{Introduction}

Hereditary Leyomiomatosis and Renal Cell Cancer (HLRCC) is an autosomal dominant genodermatosis of variable penetrance associated with a mutation at the gene of fumarate hydratase $(\mathrm{FH})$ at $1 \mathrm{q}$ chromosome, in which missense mutations are the most common. This gene catalyzes the reversible hydration/dehydration of fumarate to malate in the tricarboxylic acid cycle. Those patients who are affected by biallelic mutations may suffer fumaric aciduria, progressive encephalopathy, hypotonia and epileptic seizures, and do not survive beyond months of life.

Nevertheless, those patients who present a mutation in one of their allele could develop multiple cutaneous piloleiomyomas, fibroids and have a 15\% accumulated risk of type II papillary renal cell carcinoma. High concentrations of fumarate, secondary to the deficit of $\mathrm{FH}$, carries the inhibition of the hydroxylase of hypoxia inducible factors (HIF), therefore, there is an accumulation of HIF and an increase in downstream transcription factors related to cell proliferation, cell survival and angiogenesis such as VEGF and GLUT1(1,2).

Using genomic mapping Alam et al, described a minimum interval that contained the locus of the gene, which they called MCUL1 (multiple cutaneous and uterine leiomyomata), a region of $14 \mathrm{cM}$, that can act as a tumor suppressor gene(3). To date, three large series of cases have been carried out analyzing their mutations(4-6). Gardie's study identified up to 32 mutations for the FH gene, including 21 mutations not previously described.

Recently, Shuch et al (2020) studied data sets from the 1000 Genomes Project (1000GP) and the Exome Aggregation Consortium (ExAC) containing sequencing data for the FH gene. They concluded that FH alterations are carried by 1 in 1000 patients with 
renal cell cancer and the lifetime kidney cancer penetrance its of $1.7 \%$ to $5.8 \%$ in ExAC dataset, lower than estimated(7).

\section{Case Report}

An 18-year-old man, with generalized epilepsy treated with lacosamide $100 \mathrm{mg} / 12 \mathrm{~h}$ and family history of a grand father and an uncle dead by renal cancer both at age of 40 , starts with monosymptomatic haematuria and loss of $18 \mathrm{~kg}$ of weight in 3 months. The patient is diagnosed of a mass at the upper pole of the right kidney with a size of $10.5 \times 6$ $\times 9 \mathrm{~cm}$, a retroperitoneal conglomerate of $7 \times 3 \mathrm{~cm}$ with inferior vena cava infiltration, thrombus at the right renal artery and an adenopathy at the aortic bifurcation of $4.6 \times 2.8$ cm [Image 1].

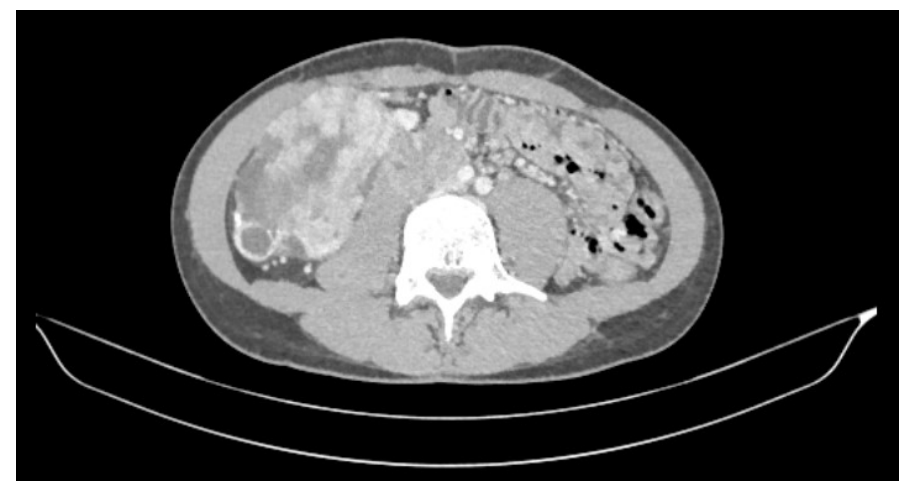

Image 1: CT at diagnosis I Mass at the upper pole of the right kidney

After confirming tumor malignancy by stereotactic biopsy with a result of papillary and tubular renal carcinoma, it is followed by a radical right nephrectomy, retroperitoneal, retrocaval and periaortic lymphadenectomy. The pathological result shows a renal cell carcinoma with papillary, tubular, tubulocistyc and solid pattern, affected vascular margin and with suspicion of fumarate hydratase (FH) loss of expression because of presence of eosinophilic nucleolus surrounded by a perinuclear halo. TNM stage: pT3a pN1 (4 out of 7 lymph nodes affected).

After recovery it is performed a PET-CT with disease progression at lateroconal fascia, right psoas, peritoneum, retroperitoneum and a metastatic lesion at hepatic VII segment [Image 2]. The patient is also derived to genetic counseling confirming a deletion of $3.9 \mathrm{kB}$ at $1 \mathrm{q} 43$ chromosomal region, which includes exon 8 of the FH gen in heterozygosity, associated with hereditary leiomyomatosis and renal cell cancer (HLRCC). 


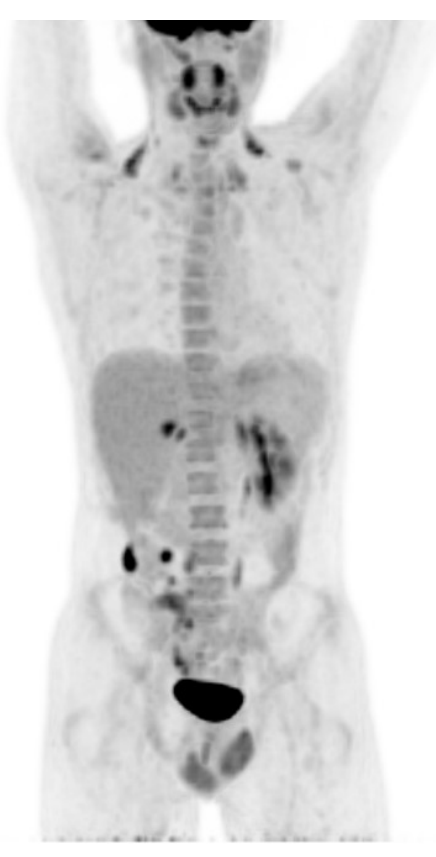

Image 2: PET-CT confirming first progression, with locoregional lesions, hepatic and retroperitoneal metastasis

The patient starts biweekly bevacizumab $(10 \mathrm{mg} / \mathrm{m} 2)$ and erlotinib $150 \mathrm{mg} / 24 \mathrm{~h}$. He develops grade 2 hypothyroidism, which is treated with supplementation of oral levothyroxine and grade 3 acneiform rash requiring tyrosine-kinase inhibitor (TKI) interruption and topic treatment. In first reevaluation after 2 months patient presents radiological disease progression by RECIST 1.1 criteria with retroperitoneal node increase up to $32 \mathrm{x}$ $24 \mathrm{~mm}$ and a new node anterior to the right psoas of $23 \times 16 \mathrm{~mm}$ [Image 3]. With ECOG-PS 1 , mild abdominal pain and grade 1-2 rash patient starts new treatment scheme with pembrolizumab 200mg every 3 weeks combined with axitinib 5mg/12h. After 2 months patients refer disappearance of abdominal pain and CT shows major partial response with reduction of the retroperitoneal node and resolution of the rest of disease [Image 4]. Actually, patient is in 11th cycle of treatment with maintained major partial response, with grade 1-2 diarrhea managed with loperamide and requiring temporary interruptions of axitinib.

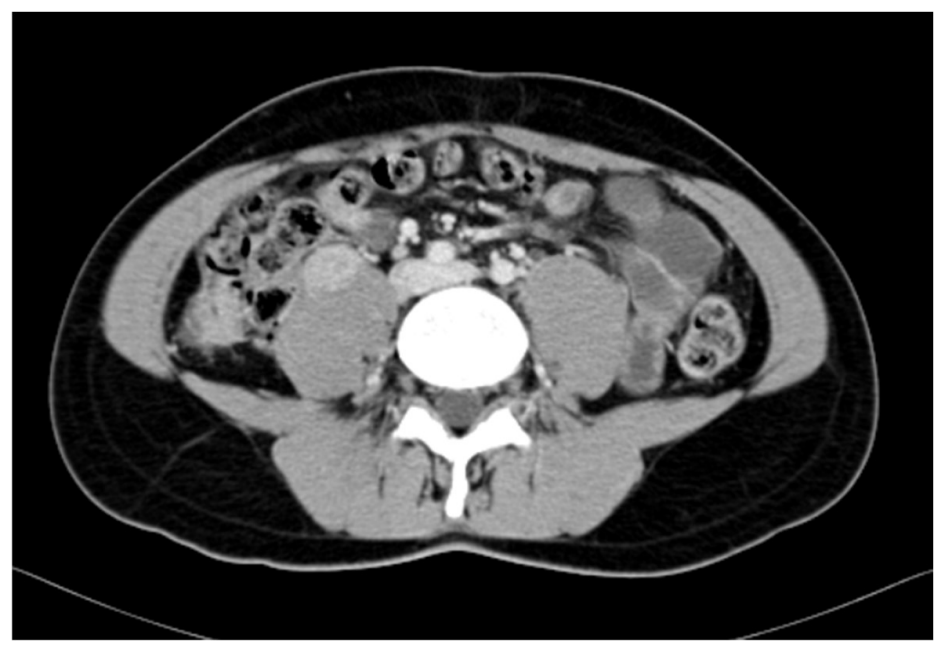

Image 3: CT confirming second progression I Increase of retroperitoneal node and a new node anterior to the right psoas 


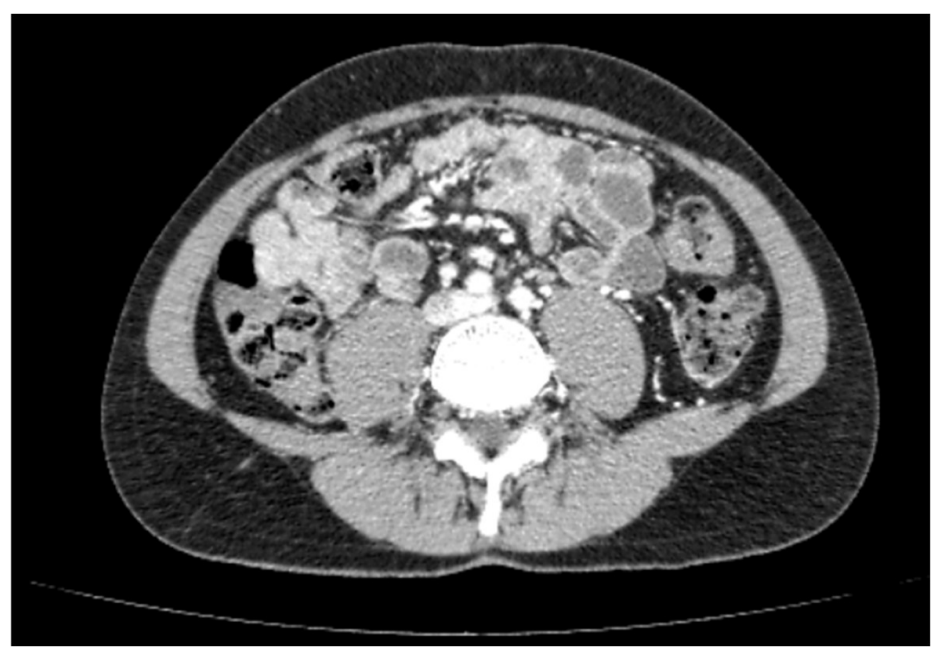

Image 4: CT with major partial response I Shows stable retroperitoneal node and resolution of the rest of metastatic disease.

Investigators obtained written consent from the patient for publishing this case report including medical history, images and treatments received.

\section{Discussion}

HLRCC is a rare disease with no more than 300 families detected around the world and that is way it is considered an orphan disease with no relevant clinical trials published. Nowadays the treatments offered are based on results of small subgroups gathered in those mentioned big trials. Based on the NCT01130519 clinical trial where 42 out of 83 patients had HLRCC, NCCN guidelines (National Comprehensive Cancer Network) suggest the combination of bevacizumab and erlotinib as first line treatment for the metastatic disease. This trial showed an overall response rate (ORR) of $72,1 \%$, with progression free survival (PFS) of 21,1 months and grade 3-4 toxicities of $47 \%(8)$.

In the Keynote426 clinical trial, they combined axitinib and pembrolizumab in RCC has encouraging results with an ORR of $59,3 \%$ and a PFS of 15,1 months independent of PDL1 expression and International Metastatic Renal Cell Carcinoma Database Consortium risk groups(9). For the use of the combination in the case reported, we relied on the pseudo-hypoxic status triggered by FH deficiency, as well as a pro-inflammatory condition demonstrated by a retrospective study in which a higher proportion of PDL1 overexpression was observed in the subgroup of patients with HLRCC, together with a greater number of PD1-expressing CD8 tumor-infiltrating lymphocytes at the tumor margins. The authors concluded that these patients could present a greater response to immune checkpoint inhibitors (ICPI)(10).

Recently, the first case of stage IV HLRCC with pleural effusion and treated with sintilimab and axitinib has been described, with disappearance of the effusion and stabilization of the renal mass(11). This would be the second case reported with an extraordinary response to a combination of ICPI and a TKI as well as a discovery of a new genomic deletion in the HLRCC syndrome.

\section{Conclusions}

This case exemplifies the need to focus on young patients affected by kidney tumors and carry out adequate genetic counseling in order to suspect specific syndromes and be 
able to make a better mutational determination. In addition, this case report brings clinicians a new effective treatment with promising results in a rare disease with few proven treatments.

Funding: This research received no external funding.

Informed Consent Statement: Informed consent was obtained from the subject involved in the case report

Conflicts of Interest: The authors declare no conflict of interest.

\section{References}

1. Maher ER. Hereditary renal cell carcinoma syndromes: diagnosis, surveillance and management. World J Urol [Internet]. 2018;36(12):1891-8. Available from: https://doi.org/10.1007/s00345-018-2288-5

2. Patel VM, Handler MZ, Schwartz RA, Lambert WC. Hereditary leiomyomatosis and renal cell cancer syndrome: An update and review. J Am Acad Dermatol [Internet]. 2017;77(1):149-58. Available from: http://dx.doi.org/10.1016/j.jaad.2017.01.023

3. Alam NA, Barclay E, Rowan AJ, Tyrer JP, Calonje E, Manek S, et al. Clinical features of multiple cutaneous and uterine leiomyomatosis: an ｕnderdiagnosed tumor syndrome. Arch Dermatol. 2005 Feb;141(2):199-206.

4. Tomlinson IPM, Alam NA, Rowan AJ, Barclay E, Jaeger EEM, Kelsell D, et al. Germline mutations in FH predispose to dominantly inherited uterine fibroids, skin leiomyomata and papillary renal cell cancer. Nat Genet. 2002 Apr;30(4):406-10.

5. Gardie B, Remenieras A, Kattygnarath D, Bombled J, Lefèvre S, Perrier-Trudova V, et al. Novel FH mutations in families with hereditary leiomyomatosis and renal cell cancer (HLRCC) and patients with isolated type 2 papillary renal cell carcinoma. J Med Genet. 2011 Apr;48(4):226-34.

6. Wei M-H, Toure O, Glenn GM, Pithukpakorn M, Neckers L, Stolle C, et al. Novel mutations in FH and expansion of the spectrum of phenotypes expressed in families with hereditary leiomyomatosis and renal cell cancer. J Med Genet. 2006 Jan;43(1):18-27.

7. Shuch B, Li S, Risch H, Bindra RS, McGillivray PD, Gerstein M. Estimation of the carrier frequency of fumarate hydratase alterations and implications for kidney cancer risk in hereditary leiomyomatosis and renal cancer. Cancer. 2020 Aug;126(16):3657-66.

8. Srinivasan R, Gurram S, Al Harthy M, Singer EA, Sidana A, Shuch BM, et al. Results from a phase II study of bevacizumab and erlotinib in subjects with advanced hereditary leiomyomatosis and renal cell cancer (HLRCC) or sporadic papillary renal cell cancer. J Clin Oncol [Internet]. 2020 May 20;38(15_suppl):5004. Available from: https://doi.org/10.1200/JCO.2020.38.15_suppl.5004

9. Rini BI, Plimack ER, Stus V, Gafanov R, Hawkins R, Nosov D, et al. Pembrolizumab plus axitinib versus sunitinib for advanced renal-cell carcinoma. N Engl J Med. 2019;380(12):1116-27.

10. Walter B, Gil S, Naizhen X, Kruhlak MJ, Linehan WM, Srinivasan R, et al. Determination of the Expression of PD-L1 in the Morphologic Spectrum of Renal Cell Carcinoma. J Cancer. 2020;11(12):3596-603.

11. Feng D, Yang Y, Han P, Wei X. The preliminary outcome of the combination of immunotherapy and targeted therapy after recurrence and metastasis for hereditary leiomyomatosis and renal cell cancer-a case report. Vol. 9, Translational andrology and urology. 2020. p. 789-93. 